Historic, Archive Document

Do not assume content reflects current scientific knowledge, policies, or practices. 



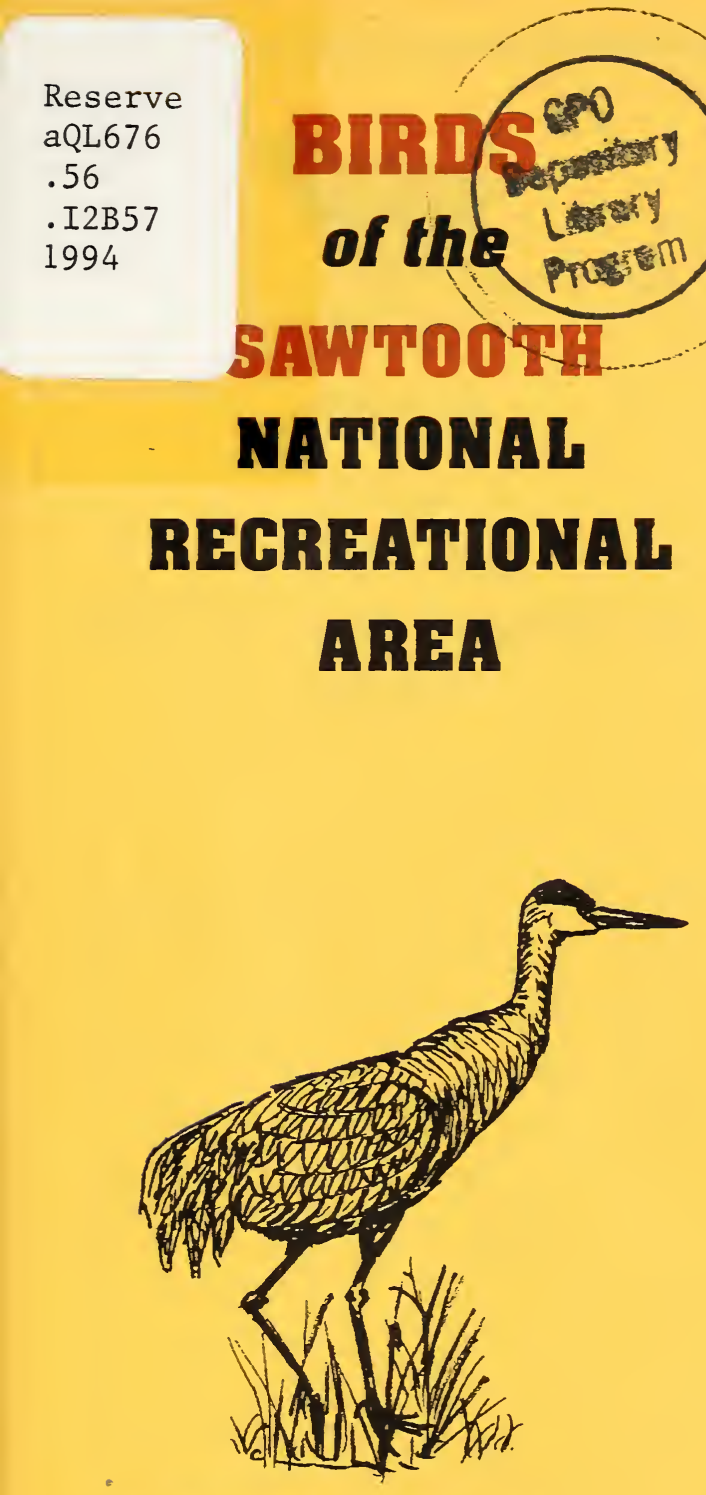

aQL676

.56

. I2B 57

1994 RECREATIONAL AREA

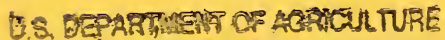
MATONAL AGRICLLTUJRAL LIBRARY

OCT IS 1994

\section{CATALOGING PREP:}

$\begin{array}{llll}\text { United States } & \text { Forest } & \text { Intermountain } & \text { Sawtooth } \\ \text { Department of } & \text { Service } & \text { Region } & \begin{array}{l}\text { National } \\ \text { Forest }\end{array}\end{array}$




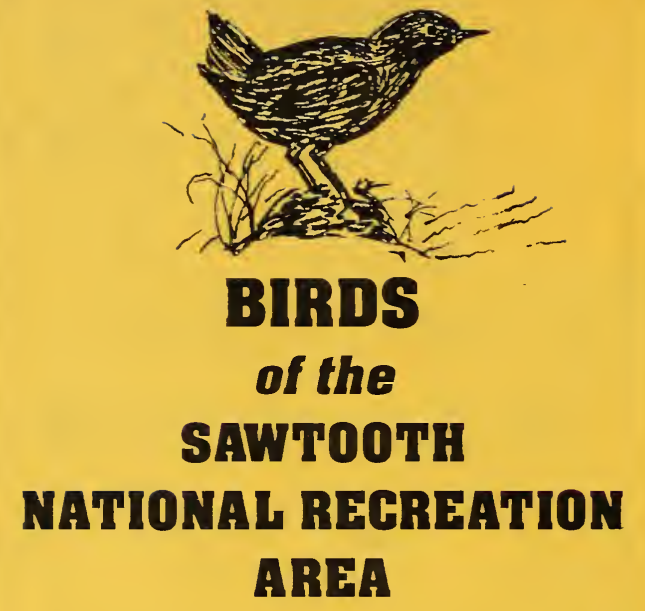

\section{INTRODUCTION}

The Sawtooth National Recreation Area (NRA), located in central Idaho, contains the headwaters of three major river systems and includes some of the most beautiful mountain ranges in the state. The famous "River of No Return," the Salmon, originates in the northern portion of the Sawtooth NRA, the South Fork of the Payette drains the Sawtooths to the west, and the Big Wood River flows south past world-famous Sun Valley ski resort. Habitats vary with elevation from ancient stands of limber pine at over 11,000 feet, through forests of Douglas-fir, subalpine fir, lodgepole pine and aspen, to grassland and sagebrush at 5200 feet. These diverse habitats provide food and shelter for approximately 214 species of birds that can be found on the Sawtooth NRA either in migration or during the nesting season.

This list is intended to be used with a field guide to help identify and observe birds during your visit to the area. If you see any species not on the list or listed as endangered, please report the sighting to the Sawtooth NRA headquarters (eight miles north of Ketchum) or the Stanley Ranger Station. Need identification help? Feel free to stop by at either location for some friendly advice. 


\section{LEGEND}

\section{Abundance}
A = Abundant or common in large numbers
$\mathrm{C}=$ Common in suitable habitat
$U=$ Uncommon (present in small numbers)
$\mathrm{O}=$ Occasionally seen
$\mathrm{R}=$ Rare (recorded less than 5 times)

\section{Habitat}

1 = Lakes

2 = Marshes

3 = Riparian areas / rivers and streams

$4=$ Grasslands $/$ meadows

5 = Sagebrush / brushlands

$6=$ Coniferous forests

7 = Aspen / cottonwood forests

$8=$ Rocky areas and cliffs

9 = Buildings, towns

$10=$ Alpine rock and meadow

\section{Season}

$S$ = Spring-March to May

$S$ = Summer-June to August

$\mathrm{F}=$ Fall-September to November

$\mathrm{W}=$ Winter-December to February

Species in bold are endangered, threatened, or sensitive (a species exhibiting a downward trend in population or habitat loss). Please report any sightings of these species to the Sawtooth NRA Headquarters or the Stanley Ranger Station. 


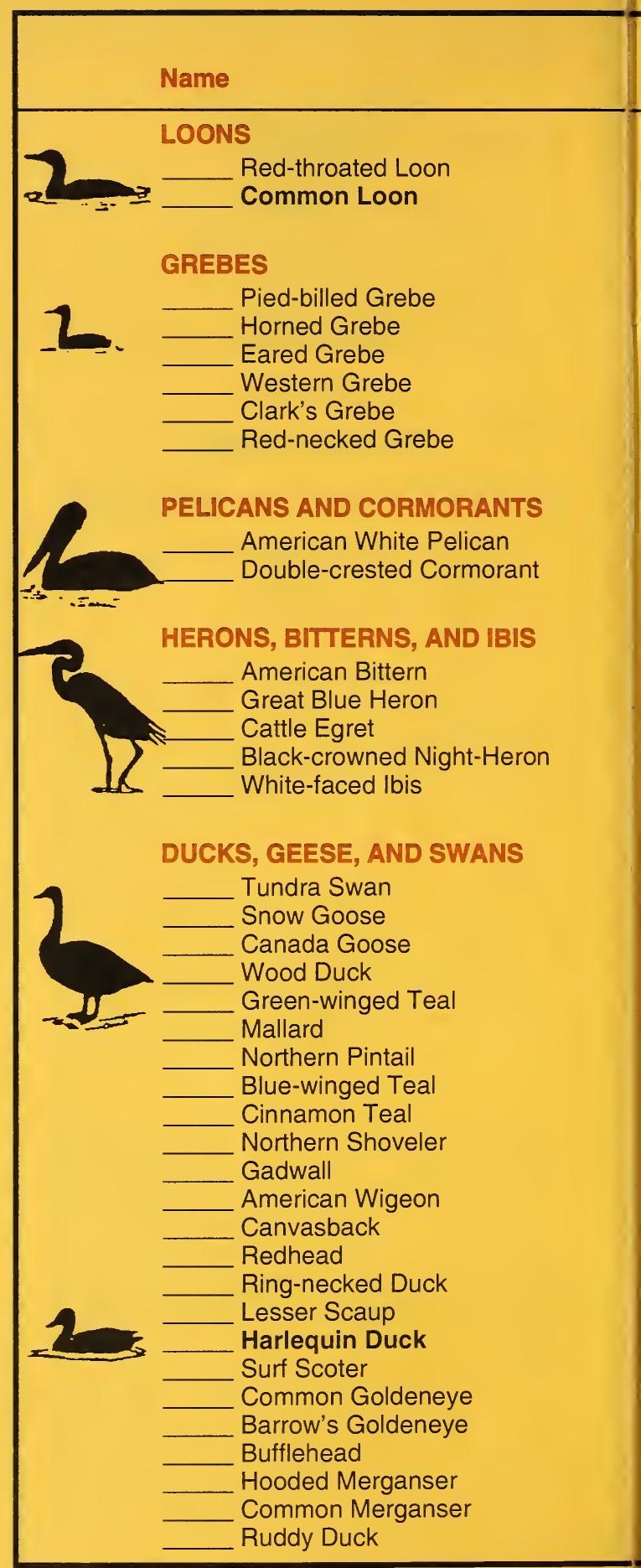





\section{Name}

VULTURES

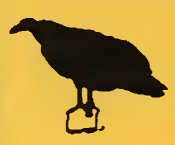

Turkey Vulture

EAGLES, HAWKS, AND FALCONS Osprey

\section{Bald Eagle}

Northern Harrier
Sharp-shinned Hawk Cooper's Hawk Northern Goshawk Swainson's Hawk Red-tailed Hawk Ferruginous Hawk

i'h Rough-legged Hawk Golden Eagle American Kestrel Merlin Peregrine Falcon Prairie Falco

GROUSE, PARTRIDGES, AND QUAIL

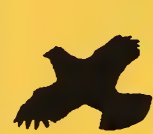

Chukar Spruce Grouse Blue Grouse Ruffed Grouse Sage Grouse Turkey

RAILS, COOTS, AND CRANES

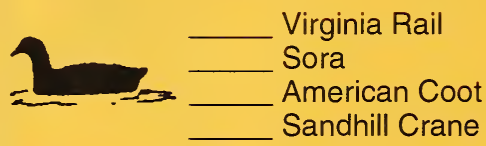

\section{PLOVERS}

Semipalmated Plover

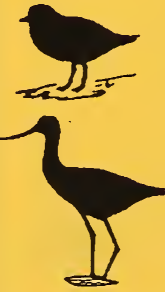
Killdeer

\section{STILTS AND AVOCETS} Black-necked Stilt American Avocet

\section{SANDPIPERS}

Greater Yellowlegs Lesser Yellowlegs Solitary Sandpiper Willet Spotted Sandpiper 


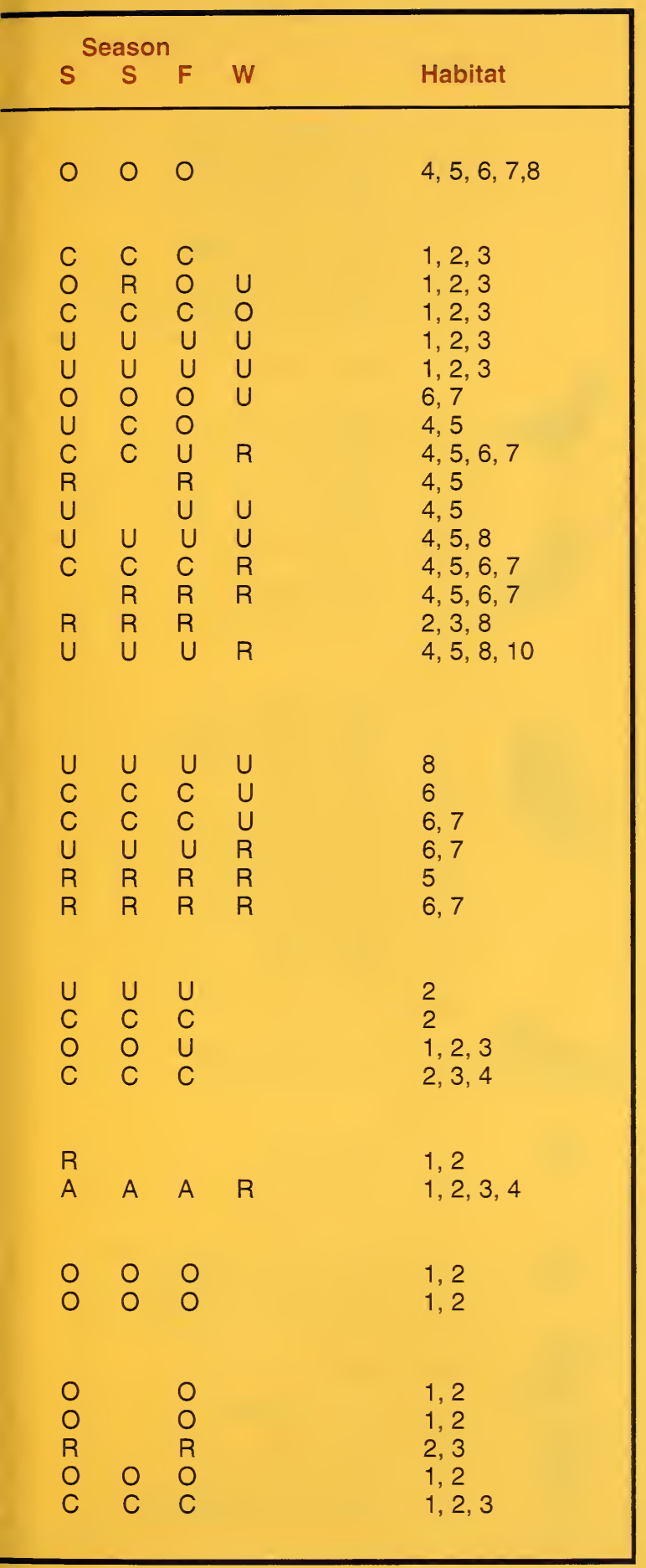


Name

Long-billed Curlew Western Sandpiper Least Sandpiper Baird's Sandpiper Pectoral Sandpiper Common Snipe Wilson's Phalarope Red-necked Phalarope

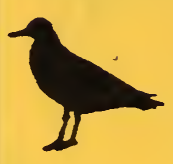

GULLS AND TERNS

Franklin's Gull Ring-billed Gull California Guli Caspian Tern Forster's Tern

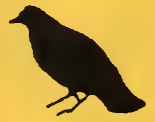

\section{PIGEONS AND DOVES}

Rock Dove Mourning Dove

\section{OWLS}

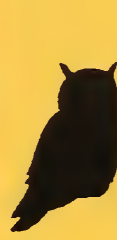
Barn Owl Flammulated Ow Western Screech Owl Great Horned Owl Snowy Owl Northern Pygmy-Owl Great Gray Owl Long-eared Owl Short-eared Owl Boreal Owl Northern Saw-whet Owl

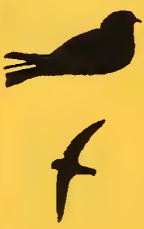

GOATSUCKERS

Common Nighthawk Common Poorwill

SWIFTS

Black Swift Vaux's Swift

HUMMINGBIRDS

Black-chinned Hummingbird

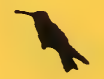
Calliope Hummingbird Broad-tailed Hummingbird

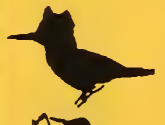
Rufous Hummingbird

\section{KINGFISHERS}

Belted Kingfisher

WOODPECKERS 


\begin{tabular}{|c|c|c|c|c|}
\hline \multicolumn{3}{|c|}{ Season } & w & Habitat \\
\hline$U$ & $R$ & $U$ & & 2,4 \\
\hline $\mathrm{R}$ & & $\mathrm{R}$ & & 1,2 \\
\hline $\mathrm{R}$ & $\mathrm{R}$ & $\mathrm{R}$ & & 1,2 \\
\hline & $\mathrm{R}$ & $\begin{array}{l}R \\
R\end{array}$ & & \\
\hline C & C & C & $U$ & 2 \\
\hline C & C & 0 & & 1,2 \\
\hline $\mathrm{R}$ & & $\mathrm{R}$ & & 1,2 \\
\hline $\mathrm{R}$ & & & & $1,2,3$ \\
\hline C & C & 0 & & $1,2.3$ \\
\hline C & C & C & & $1,2,3$ \\
\hline$R$ & $\mathrm{R}$ & $\mathrm{R}$ & & 1,23 \\
\hline $\mathrm{R}$ & $\mathrm{R}$ & $\mathrm{R}$ & & $1,2,3$ \\
\hline 0 & 0 & 0 & 0 & 8,9 \\
\hline 0 & $U$ & $U$ & & $4,5,6,7$ \\
\hline$R$ & $\mathrm{R}$ & $R$ & & $2,4,9$ \\
\hline$R$ & $\mathrm{R}$ & & & 6,7 \\
\hline $\mathrm{R}$ & $R$ & $R$ & & 3 \\
\hline$U$ & U & U & $U$ & $3,4,5,6,7,8,9$ \\
\hline & & & $\mathrm{R}$ & 2,4 \\
\hline$U$ & $U$ & $U$ & 0 & 6,7 \\
\hline $\mathrm{R}$ & $\mathrm{R}$ & $R$ & $R$ & 6,7 \\
\hline 0 & 0 & 0 & & $3,6,7$ \\
\hline 0 & 0 & 0 & & 2,4 \\
\hline $\mathrm{R}$ & $\mathrm{R}$ & $\mathrm{R}$ & $\mathrm{R}$ & 6,7 \\
\hline U & $U$ & U & $U$ & 6,7 \\
\hline U & C & C & & $4,6,7$ \\
\hline $\mathrm{R}$ & 0 & & & $6,7,8$ \\
\hline$R$ & $\mathrm{R}$ & & & 8 \\
\hline 0 & 0 & & & 6,7 \\
\hline$U$ & $U$ & 0 & & 3,4 \\
\hline C & $\mathrm{C}$ & $\mathrm{U}$ & & 3,4 \\
\hline C & C & U & & $5,6,7$ \\
\hline 0 & C & C & & $5,6,7$ \\
\hline$U$ & $U$ & $U$ & $U$ & $1,2,3$ \\
\hline C & C & C & & 3 \\
\hline C & C & C & & 6,7 \\
\hline
\end{tabular}


Red-naped Sapsucker Williamson's Sapsucker Downy Woodpecker Hairy Woodpecker Three-toed Woodpecker Black-backed Woodpecker Northern Flicker Pileated Woodpecker

\section{FLYCATCHERS} Olive-sided Flycatcher Western Wood Pewee Willow Flycatcher Hammond's Flycatcher Cordilleran Flycatcher Say's Phoebe Western Kingbird Eastern Kingbird

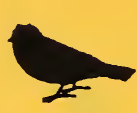

\section{LARKS}

Horned Lark

\section{SWALLOWS}

Tree Swallow

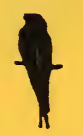

Violet-green Swallow Northern Rough-winged Swallow Bank Swallow Cliff Swallow Barn Swallow

JAYS, MAGPIES, AND CROWS

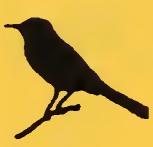
Gray Jay Steller's Jay Clark's Nutcracker Black-billed Magpie American Crow Common Raven

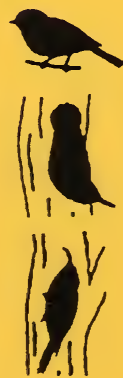

\section{CHICKADEES}

Black-capped Chickadee Mountain Chickadee

\section{NUTHATCHES}

Red-breasted Nuthatch White-breasted Nuthatch

\section{CREEPERS}

Brown Creeper 
Season

$S$ S F W

Habitat

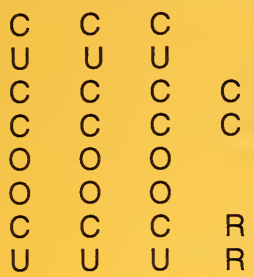

6,7

6

6,7

6,7

6

6

$3,6,7$

6,7

$\begin{array}{ll}U & U \\ C & C\end{array}$

6

U U

C C

C C

O 0

$U$
$U$

$3,6,7$

3

6

6,7

5,8

3,5

3

O O O R

4,5

$\begin{array}{lll}\text { C } & \text { c } & \text { O } \\ \text { C } & \text { C } & \text { O }\end{array}$

1,$2 ; 3$

$1,2,3$

$1,2,3$

$1,2,3$

$1,2,3,8$

$1,2,3,9$

$\begin{array}{lll}\text { A } & \text { A } & \text { O } \\ \text { C } & \text { C } & \text { O }\end{array}$

$U \quad U \quad U \quad U$

6

$\begin{array}{llll} & U & U & U\end{array}$

C C C C

C C C C

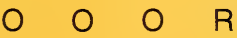

$\cup \quad \mathrm{C} \quad \mathrm{C}$

6

6

$3,5,7$

$6,7,9$

$4,5,6,7,8$

$\begin{array}{llll}C & C & C & C \\ C & C & C & C\end{array}$

6,7

6

$\begin{array}{llll}C & C & C & C \\ U & U & U & U\end{array}$

6,7

6,7

$U U U U$

6,7 


\section{Name}

\section{WRENS}

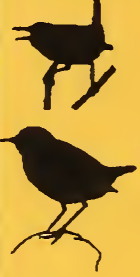

Rock Wren Canyon Wren House Wren Winter Wren

\section{DIPPERS}

_ American Dipper

THRUSHES

Golden-crowned Kinglet Ruby-crowned Kinglet Mountain Bluebird Townsend's Solitaire Swainson's Thrush Hermit Thrush American Robin Varied Thrush

THRASHERS Gray Catbird Sage Thrasher

WATER PIPITS American Pipit

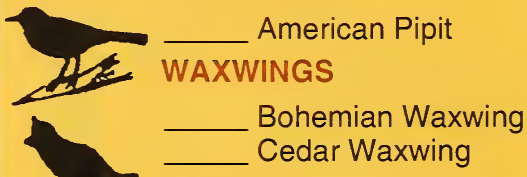

\section{SHRIKES}

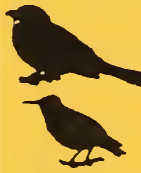

Northern Shrike Loggerhead Shrike

\section{STARLINGS}

European Starling

\section{VIREOS}

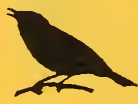

Solitary Vireo

Warbling Vireo

WOOD WARBLERS

Orange-crowned Warbler Nashville Warble

Y Yellow Warbler Yellow-rumped Warbler Townsend's Warbler Northern Waterthrush MacGillivray's Warbler Common Yellowthroat Wilson's Warbler

\section{TANAGERS}




\section{Season}

$\begin{array}{lll}U & U & U \\ O & O & 0\end{array}$

8

C C U R

O O O O

6,7

6

C C C C

3

$\begin{array}{llll}0 & 0 & 0 & 0\end{array}$

6

C C C O

C C C

6,7

C C C O

C C C

$4,5,7$

$5,6,10$

6,7

C C C

6,7

A $C$ C $C$ O

U O O

$3,4,5,6,7$

6

$\begin{array}{lll}U & U & U \\ O & O & 0\end{array}$

3,5

5

O U O

$3,4,10$

O R U U

O O O R

$5,6,7$

$5,6,7$

$\begin{array}{llll}U & 0 & 0 & U\end{array}$

$5,6,7$

$5,6,7$

C C C O

9

$\begin{array}{lll}\text { O U O } & \text { U } & 0\end{array}$

6,7

$3,6,7$

$\begin{array}{lll}O & 0 & 0\end{array}$

$3,6,7$

R R

$5,6,7$

C C C

3

6,7

$U$ U U

R R R

6

3

C C U 3

$U U U$

2, 3

$U U U$

3

C C C

6,7 
GROSBEAKS, SPARROWS, AND BUNTINGS

Rose-breasted Grosbeak

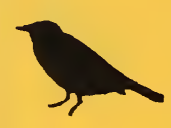
Black-headed Grosbeak Lazuli Bunting Green-tailed Towhee Rufous-sided Towhee American Tree Sparrow Chipping Sparrow Brewer's Sparrow Vesper Sparrow Lark Sparrow Sage Sparrow Savannah Sparrow Fox Sparrow Song Sparrow

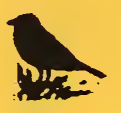
Lincoln's Sparrow White-crowned Sparrow Harris' Sparrow Dark-eyed Junco Snow Bunting

BLACKBIRDS, MEADOWLARKS, AND ORIOLES

Red-winged Blackbird

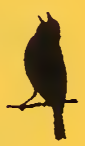
Western Meadowlark Yellow-headed Blackbird Brewer's Blackbird Brown-headed Cowbird Northern Oriole

\section{FINCHES}

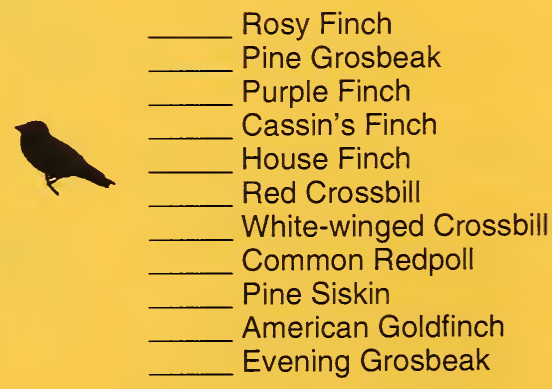




\begin{tabular}{|c|c|c|c|c|}
\hline$s$ & $\begin{array}{c}\text { asc } \\
\text { S }\end{array}$ & ${ }^{n} F$ & w & Habitat \\
\hline $\begin{array}{l}C \\
C \\
C \\
\text { O } \\
R \\
C \\
\text { U } \\
C \\
C \\
C \\
C \\
R \\
A\end{array}$ & $\begin{array}{l}C \\
C \\
C \\
O \\
\text { R } \\
C \\
U \\
C \\
C \\
C \\
C\end{array}$ & $\begin{array}{l}R \\
C \\
C \\
O \\
U \\
O \\
U \\
C \\
C \\
O \\
R \\
U \\
U \\
C \\
U \\
C \\
R \\
C \\
R\end{array}$ & $\begin{array}{l}R \\
O \\
U\end{array}$ & $\begin{array}{l}6,7 \\
3,6,7 \\
3,6,7 \\
5 \\
3,5 \\
3,5 \\
5 \\
5 \\
5 \\
5 \\
5 \\
2,4 \\
3,7 \\
2,3 \\
2,3 \\
5,6,7 \\
5,6,7 \\
5,6,7 \\
4\end{array}$ \\
\hline $\begin{array}{l}A \\
C \\
O \\
A \\
\text { C } \\
\text { U }\end{array}$ & $\begin{array}{l}A \\
C \\
O \\
A \\
C \\
U\end{array}$ & $\begin{array}{l}A \\
C \\
O \\
A \\
C \\
\text { U }\end{array}$ & $\begin{array}{l}R \\
R\end{array}$ & $\begin{array}{l}2,3 \\
4,5 \\
2 \\
2,3,4,5,6,7 \\
5,6,7 \\
3,7\end{array}$ \\
\hline $\begin{array}{l}\text { U } \\
\text { O } \\
\text { R } \\
A \\
\text { R } \\
\text { C }\end{array}$ & $\begin{array}{l}C \\
U \\
U\end{array}$ & $\begin{array}{l}C \\
U \\
U\end{array}$ & $\begin{array}{l}U \\
O \\
U \\
C \\
O\end{array}$ & $\begin{array}{l}4,8,10 \\
6 \\
6,7 \\
6 \\
5,7 \\
6,7 \\
6,7 \\
5,6,7 \\
6,7 \\
3,5,7 \\
6,7\end{array}$ \\
\hline 0 & 0 & 0 & 0 & 9 \\
\hline
\end{tabular}


The following species are hypothetical or accidental. They do not normally occur on the Sawtooth NRA.

\section{ACCIDENTALS}

Gyrfalcon

Bonaparte's Gull

Black Tern

White-headed Woodpecker

Dusky Flycatcher

Chestnut-backed Chickadee

Marsh Wren

Western Bluebird

Red-eyed Vireo

Yellow-throated Vireo

Lark Bunting

Great-tailed Grackle

Common Grackle

\section{ACKNOWLEDGEMENTS}

This list contains birds seen and recorded by Forest Service personnel, as well as Idaho Department of Fish and Game personnel and local birdwatchers.

Many thanks to Larry Barnes, Robin Garwood, Gary Gadwa, Cindy Hagas, Hadley Roberts, Louise Stands, Brian Sturges, and Jenny Taylor for their input and review of this list. Thanks to the Los Angeles Audubon Society for use of the bird silhouettes. Also, a special thanks to Louise Stands for allowing the use of her drawings of the American dipper and the sandhill crane. 
4,5

1, 2, 3

2,3

6

5,7

6,7

2

$4,5,7$

$5,6,7$

6

5

4, 9

6,7

\section{NOTES}




\section{NOTES}




\section{NOTES}


NATIONAL AGRICULTURAL LIBRARY

||||||||||||||||||||||||||||||||||||||||||||||||||||||||||

|| ||||||||||||||||||||||||||||||||||||||||||||||||||||| 1022442948

26.23.414.05/92-Revised/Reprinted 1994. 